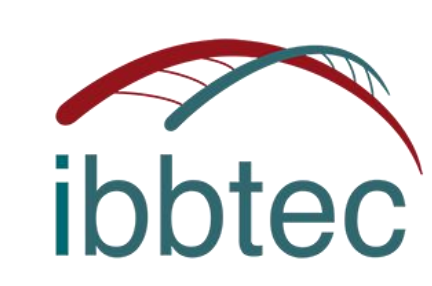

\title{
EFFECT OF MTOR SILENCING IN MOUSE INFRALIMBIC CORTEX ON DEPRESSIVE-LIKE BEHAVIOUR
}

P.2.a.010

\author{
E. Garro-Martinez $z^{1,2,3}$, N. Fullana ${ }^{3,4}$, V. Paz ${ }^{3,4}$, E. Ruiz ${ }^{3,4}$, A. Bortolozzi ${ }^{3,4}$, A. Pazos ${ }^{1,2,3}$, F. PilarCuellar ${ }^{1,2,3}$
}

IInstitute of Biomedicine \& Biotechnology of Cantabria (IBBTEC) (UC-CSIC-SODERCAN), Santander, Spain 2University of Cantabria, Department of Physiology and Pharmacology, Spain.

${ }^{3}$ CIBERSAM, Biomedical Research Networking Center Consortium, Mental Health, Carlos III Institute, Spain.

${ }^{4}$ Department of Neurochemistry and Neuropharmacology, Institut d'Investigacions Biomèdiques de Barcelona, CSIC - IDIBAPS.

\section{INTRODUCTION}

Among the hypotheses postulated with the aim of clarifying the neurobiological basis of major depressive disorder (MDD), the neurotrophic hypothesis is one of the most accepted [1]. The extremely important role of trophic factors such as the brain derived neurotrophic factor (BNDF) in both, the etiopatology and the antidepressant effect, is well known [2]. However, in recent decades, the intracellular pathways related to neural proliferation and plasticity have gained strength in the field [3]. In this regard increased activation of mammalian target of rapamycin (mTOR) pathway in the medial prefrontal cortex (mPFC), is necessary for the rapid antidepressant effect of the $\mathrm{N}$ Methyl-D-aspartate (NMDA) antagonist ketamine [4].

\section{AIM OF THE STUDY}

In an attempt to characterize the role of mTOR pathway in the neurobiology of this mood disorder, we silenced the expression of $\mathrm{mTOR}$ in MPFC, specifically in the infralimbic and prelimbic cortices (IL and PL), since the modulation of neuronal activity in the IL underlies the antidepressant and anxiolytic actions of ketamine [5].
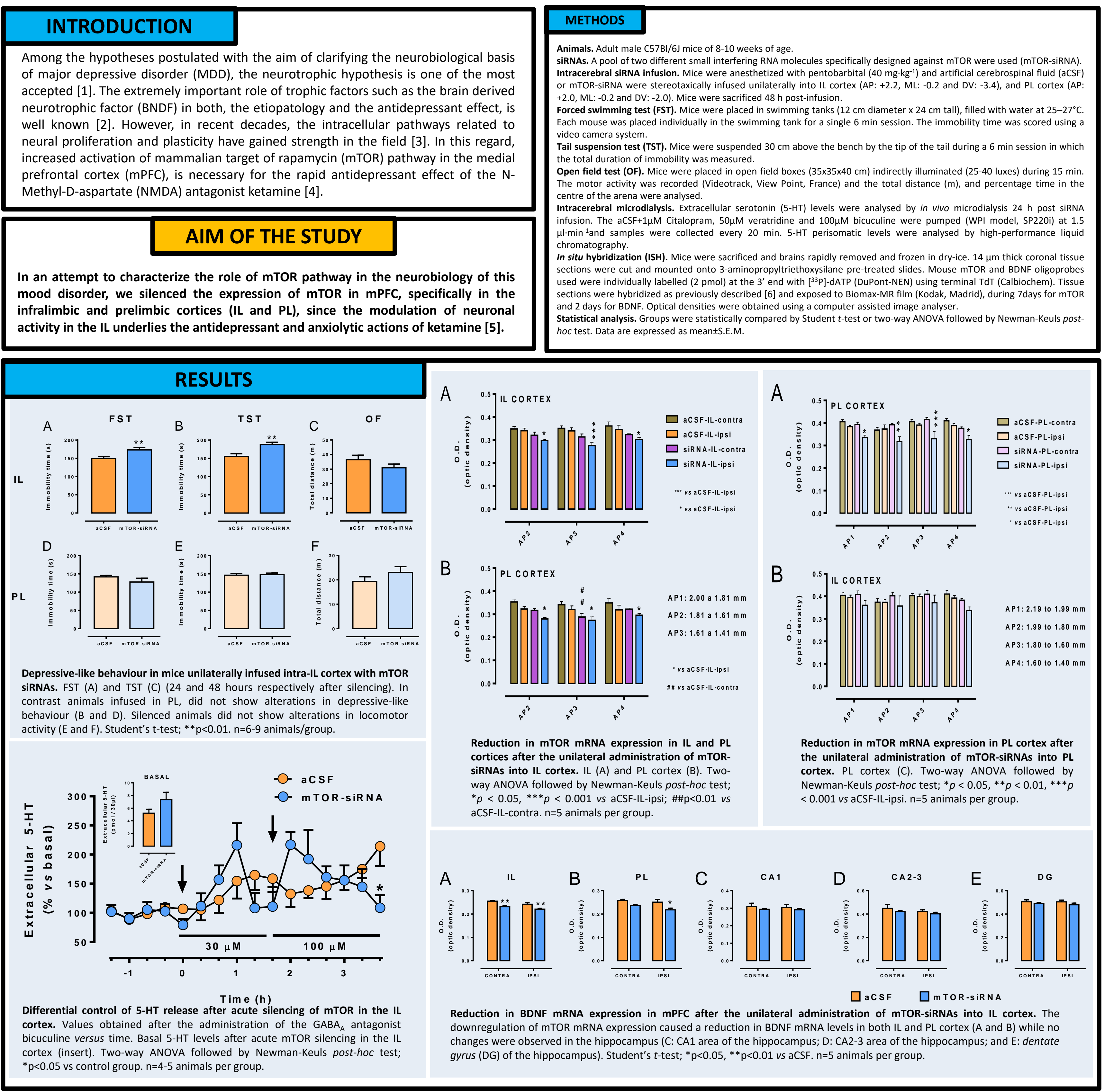

\section{CONCLUSIONS}

The present study suggests that the mTOR pathway in IL cortex, but not in PL cortex, plays a major role in the depressive-like behaviour. This allows a better understanding of the biological basis of MDD and new approaches to antidepressant drug discovery.

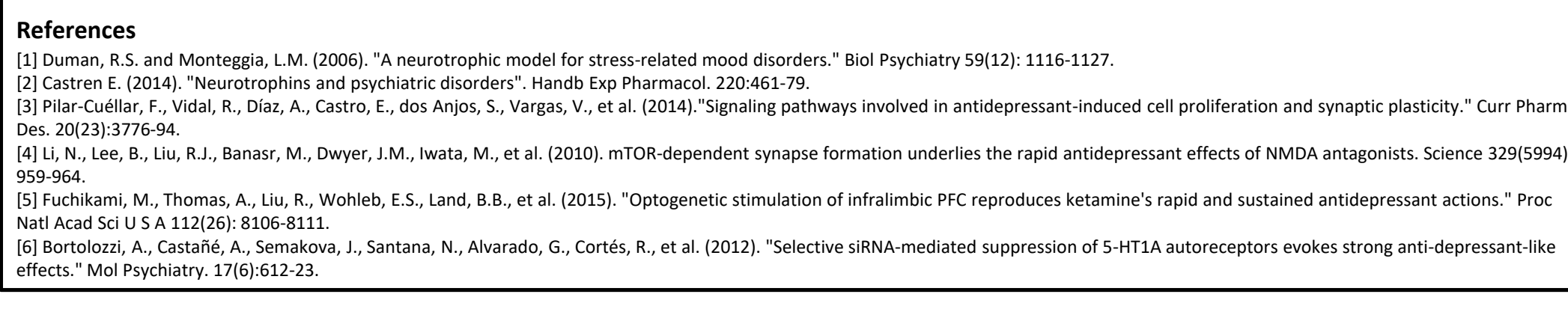

